\title{
Il ruolo della sensibilità interculturale nella didattica contemporanea
}

\author{
Anja Zorman \\ Università del Litorale, Slovenia \\ anja.zorman@fhs.upr.si
}

Il mondo della scuola si trova continuamente alle prese con le sfide che originano nella società, a suo turno in continua evoluzione e trasformazione. La globalizzazione, le migrazioni rendono la società sempre più linguisticamente e culturalmente eterogenea. La scuola ha tra l'altro il compito di educare alla convivenza, e al mutuo rispetto tra allievi di diversa origine linguistico culturale. Tuttavia, negli insegnanti, e nelle persone in generale, si nota spesso una contraddizione tra la definizione dell'educazione interculturale e dei suoi obiettivi e l'effettiva condotta di queste persone nel contatto con individui che percepiscono linguisticamente, culturalmente, etnicamente diversi (Gorski 2008). La scuola dovrebbe fare in modo di avvicinare tra loro gli allievi (e gli insegnanti) e di creare un clima in cui tutti possano trarre beneficio l'uno dall'altro. A tal proposito dovrebbe abbandonare l'idea di costruire una cultura comune, che presuppone inevitabilmente la denigrazione di determinate culture e la glorificazione di altre. La comunicazione interculturale dovrebbe invece partire dall'individualità di ciascun allievo. Pertanto, gli insegnanti ed altre figure operanti nel settore devono possedere un alto livello di sensibilità interculturale e chiare identificazioni linguistico culturali. Solo in questo modo saranno in grado di condurre un'educazione interculturale che si basi sulla promozione reciproca della comprensione tra persone, sullo sviluppo delle capacità di entrare concettualmente nel mondo dell'altro e quindi di provare empatia per gli interlocutori e generare una reciproca comprensione. Se non si sviluppa la sensibilità interpersonale e interculturale tra allievi di diversa origine culturale e/o linguistica è probabile che da adulti adotteranno schemi di comportamento determinati da visioni del mondo monoculturale, rifacendosi alla cultura a cui sono stati maggiormente esposti durante la vita (Spiteri 2017).

Parole chiave: didattica, educazione interculturale, sensibilità interculturale, insegnanti, allievi

(cc)BY-SA https://doi.org/10.26493/978-961-293-045-5.15-29

\section{Introduzione}

Nonostante non ci sia ancora una definizione dell'educazione interculturale universalmente condivisa (Arasaratnam e Doerfel 2005) e non ci sia ancora consenso su come misurare la competenza interculturale (Wahyu- 
di 2016) crediamo che le diverse definizioni in merito siano riconducibili a una base comune: il rapporto con l'altro. Nel discorso sull'interculturalità e sull'educazione interculturale l'altro è rappresentato da colui che ha una diversa origine linguistico culturale dall'ambiente ospite. Il rapporto che si ha con l'altro traspare da qualsiasi interazione con altre persone, indipendentemente dalla provenienza e/o appartenenza linguistico culturale, dal genere, dall'età, dalle convinzioni politiche o religiose ecc. Una persona intollerante nei riguardi delle persone di diversa provenienza linguistico culturale o viceversa eccessivamente entusiasta delle culture d'élite, può esibire lo stesso atteggiamento anche in altre circostanze. Dal Rapporto Europeo sull'Intolleranza, sul Pregiudizio e sulla Discriminazione (Zick, Küpper e Hövermann 2011, 67) emerge un rapporto di correlazione tra i pregiudizi verso gli immigranti, gli Ebrei, le persone di colore, i musulmani, le donne e gli omosessuali, che si evolve, secondo gli autori, nella sindrome di ostilità focalizzata su un determinato gruppo.

In questo senso, nelle aree con presenza di minoranze storiche, il delicato equilibrio tra le diverse etnie non sembra essere affetto dall'arrivo della nuova immigrazione: chi in passato si è dimostrato intollerante verso la minoranza, non diventa tollerante nei suoi confronti a causa della presenza dell'altro ancora più diverso linguisticamente e culturalmente di quello a cui è abituato. È probabile, invece, che si dimostri intollerante o addirittura più intollerante anche nei suoi confronti.

La differenza che si nota nella società, almeno in Slovenia, è lo spostamento del focus nel discorso sull'interculturalità, in particolare sull'educazione interculturale a scuola. L'azione didattica è quasi esclusivamente orientata verso l'integrazione di allievi stranieri, provenienti da famiglie migranti, prestando poca o nessuna attenzione alla presenza della minoranza storica nel nostro ambiente. Gli scambi di docenti e allievi a tutti i livelli del sistema educativo, compresi gli studenti, futuri insegnanti, avvengono a livello internazionale, spesso come attività programmate all'interno di progetti internazionali. È invece lasciata alla buona volontà del docente o del dirigente della scuola l'organizzazione di visite a enti pubblici che si occupano del mantenimento e dello sviluppo della cultura e dell'etnia italiana sul Litorale sloveno. L'altro proveniente dai territori e dalle culture lontane è decisamente più riconoscibile della minoranza, anche dall'aspetto fisico. L'altro «lontano» non può essere ignorato, richiede l'attenzione della scuola e del sistema scolastico in generale, spostando l'interesse per la minoranza in secondo piano. La mancata considerazione e il mancato rispetto per la lunga tradizione nell'educazione bilingue e pluriculturale 
di questo territorio ${ }^{1}$ sono senza dubbio in parte responsabili del calo di interesse per l'apprendimento dell'italiano nelle scuole della maggioranza e di conseguenza per il crescente calo nella competenza comunicativa in italiano lingua d'ambiente.

È inoltre problematico l'atteggiamento verso la diversità da una parte considerevole di docenti. Dalla ricerca che abbiamo condotto nell'anno scolastico 2012/2013 nelle scuole con alta presenza di allievi con diversa origine linguistico-culturale in sei aree pluriculturali in Slovenia e Italia, ${ }^{2}$ è emerso che un quarto di docenti interpellati non ha mai introdotto alcuna novità nel proprio insegnamento con lo scopo di adattarlo alla presenza di allievi di origine linguistico-culturale diversa da quella della scuola (sloveno o italiano) (Zorman e Zudič Antonič 2014). Se questo dato è stato registrato nelle scuole con alto grado di eterogeneità, è difficile credere che a livello nazionale, sloveno o italiano, la situazione nelle scuole in generale sia migliore. La totale indifferenza per i bisogni, le paure, le barriere linguistiche, i modelli culturali di allievi non sloveni o italiani, a seconda della scuola interpellata nella ricerca, è segno di mancato rispetto nei loro confronti, di una totale assenza di empatia e sensibilità interculturale, se non addirittura umana. Gorski (2008) infatti fa notare come la maggior parte della didattica continui a rafforzare invece di neutralizzare le attuali gerarchie sociali e politiche per cui anche docenti con le migliori intenzioni non riescono a mettere in atto un adeguato approccio all'insegnamento in classi linguisticamente ed etnicamente eterogenee. Nonostante che dalle ricerche emerga che gli insegnanti possiedono generalmente alti livelli di sensibilità interculturale, i valori più alti vanno al piacere di interagire e alla considerazione dell'interlocutore nell'interazione, sono considerevolmente più bassi i valori legati al rispetto delle differenze culturali (Segura-Robles e Parra Gonzáles 2019).

La classe è un microcosmo della società odierna in cui convive una pluralità di culture (Segura-Robles e Parra Gonzáles 2019). Nella nostra ri-

${ }^{1}$ Il modello bilingue per la scuola elementare fu istituito nel 1959 (Zudič Antonič e Zorman 2004).

${ }^{2}$ E D U K A - Educare alla diversità, progetto finanziato nell'ambito del Programma per la Cooperazione Transfrontaliera Italia-Slovenia (2007-2013) dal Fondo europeo di sviluppo regionale e dai fondi nazionali. Nella ricerca hanno collaborato istituzioni di ricerca da entrambe le parti del confine e scuole con alta presenza di allievi di lingue e culture diverse sia nella zona centrale dei due stati che lungo il confine dove da entrambi i lati operano scuole sia con lingua nazionale che con lingua della minoranza come lingua d'insegnamento. L'importante differenza tra le due realtà interessa lo studio obbligatorio della lingua dell'altra etnia nel Litorale sloveno dalla scuola elementare all'istruzione secondaria. 
cerca invece un docente su dieci, operante nella scuola in cui la diversità linguistico-culturale è data per certa in base al numero e alla distribuzione normale di allievi non sloveni (o italiani) nelle classi, ha dichiarato che nelle classi in cui insegna non sono presenti allievi con nazionalità e lingua diversa da quella della scuola (Zorman e Zudič Antonič 2014). Per il campione degli insegnanti interpellati il dato può essere soggetto ad almeno due interpretazioni. Nelle scuole della minoranza slovena in Italia, in cui la percentuale di docenti che percepiscono le loro classi come culturalmente eterogenee è nettamente la più bassa del campione, è probabile che il fenomeno sia la conseguenza della cosiddetta assimilazione silenziosa a cui è (stata) soggetta la minoranza slovena in Italia. ${ }^{3}$ Anche la seconda interpretazione è riconducibile all'assimilazione, ma in maniera diametralmente opposta a quella vissuta dagli sloveni in Italia. Essa si basa sulla presupposizione che gli insegnanti non reattivi alle differenze culturali in classe abbiano una visione del mondo monoculturale. I loro schemi mentali sono del tutto adattati al punto di vista della cultura a cui sono stati maggiormente esposti durante la vita (Spiteri 2017). Banks (2001) si riferisce a loro come ad esseri a-culturali ed a-etnici in possesso di esperienze estremamente esigue con la diversità razziale, etnica o sociale. Secondo l'autore si tratta di persone che sono convinte che razza e cultura caratterizzino gli emarginati e i diversi. Banks (2001) è infatti dell'opinione che i docenti dovrebbero sviluppare le proprie identificazioni culturali e nazionali riflessive e, come aggiunge Middleton (2002), la percezione delle proprie convinzioni sulla diversità, per poter essere in grado di agire efficacemente in classi eterogenee e sostenere allievi di varie origini culturali nello sviluppo delle loro identificazioni linguistico-culturali, nonché delle loro potenzialità accademiche.

\section{La sensibilità interculturale nella didattica}

L'attenzione per la diversità linguistico-culturale, sia per le minoranze storiche, per la seconda generazione degli immigrati, che per gli immigrati recenti, richiede la partecipazione attiva di tutti i quadri della scuola. $\grave{E}$ tutt'oggi spesso presente la convinzione che l'educazione interculturale sia compito dei docenti di lingue e di scienze umanistiche. Invece le ricerche dimostrano che i più alti livelli di competenza interculturale vengono raggiunti da allievi che frequentano scuole pervase dalla sensibilità intercul-

\footnotetext{
${ }^{3}$ Per approfondimenti si veda Susič e Sedmak (1983) e Susič (2003).
} 
turale, scuole in cui ogni spazio, ogni gesto, ogni atteggiamento manifesta il rispetto e celebra la diversità (Pederson 1997).

L'educazione interculturale non può poggiare su avvenimenti e singole attività organizzate dalla scuola o sviluppate dall'insegnante in classe occasionalmente e in maniera disorganica. Essa richiede un atteggiamento generale basato sulla sensibilità interculturale e sul rispetto reciproco. Solo un insegnante che ha raggiunto almeno il livello base etnorelativo nello sviluppo della competenza interculturale (Bennett 1993) ${ }^{4}$ è in grado di percepire e valorizzare le differenze tra le culture presenti nella classe, sviluppare la competenza interculturale degli allievi (Jokikokko 2005), nonché la crescita personale e il successo accademico di tutti gli allievi, indipendentemente dall'origine linguistico-culturale.

Fondare l'accettazione dell'altro nel proprio gruppo dominante partendo dall'insieme di valori e convinzioni della cultura dominante significa ignorare le differenze culturali che convivono in una classe (Spiteri 2017). Queste differenze però esistono e la scuola che promuove la sensibilità e la comunicazione interculturale basa la propria azione sulla comprensione di tali differenze con lo scopo di incrementare l'uguaglianza sociale nell'ambiente della scuola e nella società in generale. Essendo la scuola e la classe un microcosmo della società odierna (Segura-Robles e Parra Gonzáles 2019), potrebbe fungere da laboratorio vivo in cui educare alla convivenza e al rispetto reciproco allievi con diverse origini linguistico-culturali (Spiteri 2017).

\section{Il concetto della sensibilità interculturale}

Nel modello di Chen e Starosta (1996) la competenza comunicativa interculturale si compone di (i) sensibilità interculturale, (ii) consapevolezza interculturale e di (iii) competenza interculturale. La sensibilità interculturale costituisce la dimensione emotiva della comunicazione interculturale e concerne il desiderio e la volontà della persona di riconoscere, apprezzare e accettare le differenze tra culture. Hammer, Bennett e Wiseman (2000) distinguono tra la sensibilità interculturale intesa come capacità di riconoscere e sentire rilevanti le differenze tra culture e intesa come la competenza interculturale che si realizza nella capacità di riflettere e agire in maniera adeguata in incontri interculturali. Secondo gli autori, chi possiede livelli

${ }^{4}$ Il suo Modello dello sviluppo della sensibilità interculturale distingue principalmente tra livelli etnocentrici, orientati al modello culturale della persona, ed etnorelativi, orientati a una prospettiva globale. 
alti di sensibilità interculturale, dispone di un maggiore potenziale nell'attuazione della competenza interculturale. La seconda componente della competenza comunicativa interculturale, la consapevolezza interculturale, rappresenta la dimensione cognitiva della comunicazione interculturale, riferendosi alla capacità dell'individuo di comprendere le somiglianze e le differenze delle altre culture. La competenza interculturale, infine, costituisce la dimensione comportamentale e si riferisce alla capacità di raggiungere i propri obiettivi comunicativi nell'interazione con persone di culture diverse dalla propria.

Il modo in cui il termine interculturale $e^{5}$ si richiama alla comunicazione tra persone provenienti da diverse origini culturali è molto simile dalla maniera in cui il termine interpersonale manifesta la natura degli incontri tra persone in generale (Spiteri 2017).

Da una ricerca condotta da Arasaratnam e Doerfel (2005) su 37 studenti universitari provenienti da 15 contesti culturali diversi emerge infatti una stretta correlazione tra la competenza interculturale e la competenza nella comunicazione interpersonale generale. Le persone competenti nella comunicazione interculturale dimostrano di operare in una dimensione centrata sull'altro: sono sensibili, gentili, hanno esperienza con altre culture, hanno il desiderio e si dimostrano capaci nello studio dei temi legati all'interculturalità. Il loro stile di comunicazione è orientato sull'altro: sono aperti, possiedono ottime capacità comunicative, mostrano interesse per le differenze e sono consapevoli della diversità; il loro livello di esposizione al diverso gli permette di individuare le differenze con facilità. Le qualità di una persona competente nella comunicazione interculturale, individuate dalle autrici, si possono ricondurre a due dimensioni: quella della motivazione e quella della sensibilità. La motivazione si traduce nelle abilità di interessamento all'altro, di impegnarsi a comprendere e di porre aiuto. La sensibilità traspare invece dal livello personale che una persona adotta nella comunicazione e si osserva nella sua propensità a porre domande, nella sensibilità alle origini e al contesto da cui arriva l'altro e in una prospettiva globale da cui vede il mondo, contrapposta a quella etnocentrica (Bennett 1993) o monoculturale (Banks 2001; Spiteri 2017). Bennett (1993) spiega che le persone che hanno ricevuto una socializzazione prevalentemente monoculturale hanno normalmente accesso unicamente alla visio-

\footnotetext{
${ }^{5}$ In contrasto con il termine multiculturale che presuppone una semplice compresenza di persone di diversa origine culturale che vivono e interagiscono prevalentemente all'interno del proprio gruppo culturale (Zorman e Zudič Antonič 2019).
} 
ne del mondo propria della loro cultura e non sono in grado di interpretare e quindi sentire differenze tra la loro percezione del mondo e quella delle persone di altre culture. Il punto cruciale nello sviluppo della sensibilità interculturale, sostiene l'autore, risiede nel conseguimento della capacità di interpretare e sentire differenze culturali in maniere più complesse. Le ricerche confermano che l'esperienza con altre culture è un importante fattore nello sviluppo della sensibilità interculturale. In una ricerca, condotta da Del Vilar (2010) su studenti filippini, il grado di sviluppo della sensibilità interculturale cresceva con il numero di paesi visitati, il tempo di permanenza all'estero e con il numero di amici stranieri. L'esposizione alle altre culture, sostiene l'autrice, ha portato a un naturale sviluppo della capacità di interagire con persone provenienti da altre culture e quindi della sensibilità e competenza interculturale. Tuttavia, Bennett (1993) fa notare che lo sviluppo della competenza potenziale nelle interazioni interculturali è proporzionale all'esperienza con differenze culturali sempre più complessa e sofisticata. Un atteggiamento passivo o incurante delle differenze negli scambi interculturali non può stimolare lo sviluppo della sensibilità interculturale: il contatto con altre culture non conduce quindi necessariamente al mutamento della visione del mondo e quindi alla crescita della sensibilità interculturale. È importante, come sostiene Bennett (1993) che questo contatto sia gradualmente sempre più complesso ed elaborato.

Una buona comunicazione è caratterizzata dal rispetto, dall'approccio relazionale e dall'interessamento reciproco degli interlocutori. Il rispetto emerge dall'uso della lingua, dalla cortesia, dall'abilità di comunicare a livello dell'altro e di evitare che l'altro si senta umiliato. Le persone capaci di avvicinarsi all'altro nella conversazione sono abili nel condurre una conversazione amichevole e gentile e mostrano interesse nei confronti dell'altro, dedicando tempo all'ascolto dell'interlocutore, alla conversazione, alla conoscenza e all'espansione della propria rete sociale (Arasaratnam e Doerfel 2005). Nella ricerca di Del Vilar (2010) il livello di sensibilità interculturale si è rivelato in correlazione con il numero di adesioni ad associazioni di vario genere e con il periodo dell'adesione, suggerendo che le persone sensibili all'altro sono più capaci di costruirsi un'ampia rete sociale nella quale partecipano attivamente e con successo.

Chen e Starosta (1997) riconoscono cinque componenti chiave della sensibilità interculturale: l'autostima, l'automonitoraggio, l'apertura mentale, l'empatia, il coinvolgimento nell'interazione e l'assenza di pregiudizio.

Secondo gli autori le persone con alti livelli di sensibilità culturale solitamente possiedono anche un alto livello di autostima, ovvero la consi- 
derazione che l'individuo ha di sé stesso. Spiteri (2017) sostiene che è in grado di costruire buoni rapporti con gli altri solo chi ha un buon rapporto con sé stesso. Le persone con alto livello di autostima comunicano con facilità con gli altri senza avere paura di critiche o di riconoscere i propri errori, ma anche di dare e ricevere complimenti. Inoltre, si sentono a loro agio a condividere con altri le proprie idee ed esperienze. Hanno una buona opinione degli altri e si aspettano di essere accettati da loro. Chen e Starosta (1997) ribadiscono che nella comunicazione interculturale, in cui si innalza nelle persone lo stress psicologico, legato alle esigenze di portare a termine il compito prefissato e di stabilire rapporti con altri, l'autostima diventa una variabile importante nella valutazione delle possibilità di soddisfare i propri bisogni in una determinata situazione. Più è alto il livello di autostima, più si sentono sicuri di sé e vivono l'interazione interculturale come opportunità di comunicare in maniera diversa ed entusiasmante (Del Vilar 2010). ${ }^{6}$ Nelle interazioni interculturali le emozioni positive e il rispetto dell'altro vengono dunque potenziate sia una chiara identificazione culturale e nazionale della persona (Banks 2001) che una sua alta autostima.

Il monitoraggio e l'automonitoraggio fanno parte delle «funzioni esecutive di alto ordine che intervengono nel processo comunicativo [...] che richiedono, infatti, la capacità dell'individuo di valutare gli effetti della propria azione comunicativa» (Balconi 2008). Tali capacità permettono di adattare il proprio comportamento alle condizioni in cui si svolge la comunicazione e di mettere in atto strategie comunicative competenti (Chen e Starosta 1997). Persone con alto livello di automonitoraggio sono abili nell'interpretazione degli stati mentali degli altri, dei loro pensieri, credenze ecc. (Balconi 2008) e sono più propensi al compromesso, fanno maggiore appello all'emotività, all'ingraziamento e all'influenza di riferimento, qualità che dotano le persone della sensibilità di percepire vari segnali comunicativi e di conseguenza potenziano ulteriormente le capacità di sviluppare un insieme di strategie comportamentali da impiegare in maniera efficace in varie situazioni comunicative (Chen e Starosta 1997). Nei soggetti coinvolti nella ricerca di Del Vilar (2010) il livello delle capacità comunicative erano in stretta correlazione con il livello della loro sensibilità interculturale: i soggetti che si percepivano competenti nella comunicazione possedevano

\footnotetext{
${ }^{6}$ Nella sua ricerca il livello di autostima si è rivelato più alto nei maschi rispetto alle femmine, con differenze statisticamente significative. L'autrice interpreta questo dato come legato alla cultura filippina in cui è predominante la figura maschile.
} 
una maggiore sensibilità verso le altre culture, dimostrandosi più reattivi e attenti all'interlocutore.

Il livello di sensibilità interculturale dipende dal modo in cui le persone interpretano le differenze culturali Bennett (1993). La teoria degli schemi cognitivi, un modello di elaborazione delle informazioni in cui collaborano la percezione e la cognizione, vede questi impegnati nella percezione ed elaborazione di informazioni sociali su persone, gruppi ed eventi (Arzenšek 2012). Gli schemi cognitivi aperti permettono alle persone di spiegarsi apertamente e adeguatamente e di accettare le spiegazioni degli altri (Chen e Starosta 1997). L'apertura mentale ci permette di comprendere che una stessa realtà può avere molteplici interpretazioni e quindi di adattarci alle differenze in svariati contesti culturali.

L'empatia è la componente chiave della sensibilità interculturale. All'individuo permette di immedesimarsi nell'altro e di comprendere i suoi processi psichici. Una persona con alti livelli di sensibilità interpersonale e interculturale invia e riceve messaggi incoraggianti e rassicuranti che sono alla base di una buona comunicazione interculturale in cui gli interlocutori portano reciprocamente rispetto all'altro (Del Vilar 2010). L’empatia è dunque alla base di una manifestazione reciproca di sentimenti, dell'ascolto attivo e delle reazioni verbali che dimostrano la comprensione per i sentimenti e i pensieri dell'altro (Chen e Starosta 1997). La manifestazione di identificazione, di comprensione e di considerazione dell'altro sono quindi le componenti dell'empatia che rappresentano l'essenza della sensibilità interculturale e conducono allo sviluppo della competenza interculturale (Bennett 1993).

Il grado di coinvolgimento nell'interazione dipende dalla reattività, ricettività e interessamento all'interlocutore. Una persona reattiva, ricettiva e attenta è maggiormente in grado di ricevere e comprendere i messaggi, di prendere adeguatamente i turni nella conversazione e di iniziare e concludere uno scambio interculturale in maniera naturale e adeguata alla situazione (Chen e Starosta 1997). Alcune ricerche riferiscono che anche in persone mature con alti livelli di sensibilità interpersonale può verificarsi un senso di ansia nella comunicazione con persone di diversa provenienza linguistico-culturale, ma che questo tuttavia viene a dileguarsi nell'arco di pochi minuti. I soggetti interpellati da Del Vilar (2010) hanno dichiarato che nei momenti di ansia si sentivano meno sensibili nei confronti degli interlocutori e che con il dileguarsi dell'apprensione cresceva la loro sensibilità per l'interlocutore. Secondo l'autrice l'ansia condiziona il nostro ragionamento e può derivare dalla situazione comunicativa finora sconosciuta 
oppure dalla preoccupazione di non essere in grado di portare a termine il compito prefissato e di stabilire un buon rapporto comunicativo con l'interlocutore, dicendo qualcosa di «sbagliato» oppure agendo in maniera che questi potrebbe interpretare come offensiva.

Persone guidate nelle interazioni dal pregiudizio, invece, tendono a saltare alle conclusioni prima di informarsi adeguatamente sulla situazione in cui si trovano o addirittura senza avere alcuna esperienza diretta in tali circostanze. Anche giudizi basati su esperienze parziali in una determinata situazione possono essere affetti da pregiudizi. In base a un'esperienza negativa con una persona di una cultura l'individuo può generalizzare quest'esperienza a tutti i membri di tale cultura, creando su loro in realtà un pregiudizio. Nella comunicazione interculturale si dovrebbero evitare i pregiudizi, ascoltando apertamente e sinceramente l'interlocutore, il che genera in lui sensazioni di soddisfazione psicologica. Secondo Chen e Starosta (1997), l'assenza di pregiudizio nella comunicazione interculturale ci permette di interessarci alle differenze tra le culture, trovarvi piacere e quindi di vivere con piacere gli incontri interculturali.

Una persona con alti livelli di sensibilità interculturale è associata a concetti come eterogeneità, trasmissione o mediazione, orientamento all'altro, spirito d'osservazione, motivazione, sensibilità, rispetto, relazionalità, impegno e idoneità (Arasaratnam e Doerfel 2005).

L'educazione interculturale deve basarsi sulla promozione reciproca della comprensione tra persone. A questo proposito le persone coinvolte devono essere in grado di provare empatia per gli interlocutori, e di entrare concettualmente nel mondo dell'altro per generare una reciproca comprensione. Se l'insegnante non possiede la sensibilità interculturale e di conseguenza non è in grado di svilupparla negli allievi, l'educazione interculturale o multiculturale non può raggiungere i propri obiettivi (Spiteri 2017). Tra gli insegnanti, e le persone in generale, si nota spesso una contraddizione tra la definizione dell'educazione interculturale e dei suoi obiettivi e la loro effettiva condotta nel contatto con persone che percepiscono linguisticamente, culturalmente, etnicamente diverse (Gorski 2008). La scuola dovrebbe fare in modo di avvicinare tra loro gli allievi (e gli insegnanti) e di creare un clima in cui tutti possano trarre beneficio l'uno dall'altro. A tal proposito dovrebbe abbandonare l'idea di costruire una cultura comune, che presuppone inevitabilmente la denigrazione di determinate culture e la glorificazione di altre. La comunicazione interculturale dovrebbe invece partire dall'individualità di ciascun allievo, e dovrebbe basarsi sulla comprensione dell'altro. L'educazione interculturale dovrebbe promuove- 
re la comprensione e la sensibilità tra persone di diversa origine culturale e/o linguistica e combattere l'oppressione sociale, educando le persone o promuovendo la loro consapevolezza su ciò che è oppressivo nella società. In assenza di ciò è probabile che adotteranno schemi di comportamento determinati da visioni del mondo monoculturale, rifacendosi alla cultura a cui sono stati maggiormente esposti durante la vita (Spiteri 2017).

La formazione di futuri insegnanti nonché degli insegnanti in itinere che sia basata sulla sensibilità interculturale, sulla specificità di ogni individuo coinvolto, che sia centrata sull'azione e orientata al risultato (Spiteri 2017), e soprattutto adatta al livello di competenza interculturale di ciascun individuo (DeJaeghere e Cao 2009) a suo turno promuove notevolmente lo sviluppo della sensibilità e comunicazione interculturale degli allievi. È importante, dunque, che la formazione dei docenti sia differenziata per livelli di competenza interculturale. Gli obiettivi devono basarsi sulla attuale visione del mondo degli insegnanti e allo stesso tempo essere sfidanti, ma non troppo, per non risultare frustranti. Bennett (1993) infatti fa notare come situazioni eccessivamente sfidanti richiedono reazioni che non sono in sintonia ai bisogni della crescita personale e professionale dell'individuo. Invece di portare allo sviluppo della sua sensibilità interculturale, lo ostacolano o addirittura ne causano una regressione. Bisogna inoltre essere consapevoli dei limiti dell'intervento volto allo sviluppo della sensibilità interculturale. Dalle ricerche emerge infatti una notevole variazione nella direzione e nell'entità del mutamento della sensibilità interculturale nelle persone al termine di tali percorsi. DeJaeghere e Cao (2009) hanno dimostrato che non tutti gli insegnanti manifestano riscontri positivi ai programmi di sviluppo professionale e che spesso, quando sono positivi, i risultati dell'azione formativa sono visibili soprattutto nell'azione didattica piuttosto che nell'adattamento cognitivo delle persone.

\section{Conclusioni}

Alla base della didattica e delle interazioni all'interno della scuola dovrebbe esserci la sensibilità interpersonale in generale e in specifico, interculturale. Questo vale sempre di più per la scuola contemporanea, se vuole continuare ad essere in grado di gestire le complesse differenze culturali e personali che si verificano nell'interazione di allievi di diversa origine linguistico-culturale (Spiteri 2017). Come luogo d'incontro di lingue e culture la scuola offre opportunità di crescita personale degli allievi e degli insegnanti. Ogni interazione è un'occasione per sviluppare le capacità di 
comprensione e di interazione tra gli interlocutori, a patto che siano presenti il mutuo rispetto e un genuino desiderio di imparare, di conoscersi. Nell'ambiente pluriculturale mirare a costruire una cultura comune significherebbe porre in risalto alcune culture o una cultura a detrimento delle altre. La scuola, se vuole riuscire nell'intento di attivare appieno le potenzialità, le abilità e l'ingegno degli allievi, si dovrebbe invece focalizzare sull'individualità di ciascuno di loro. L'atteggiamento dell'insegnante verso gli allievi incide significativamente sul loro successo accademico e generale, ed è di particolare rilievo nel contatto con allievi che provengono da famiglie socialmente più deboli e da famiglie di diversa provenienza culturale. Il tratto che contraddistingue gli insegnanti in grado di lavorare con successo nelle classi culturalmente e linguisticamente eterogenee è la sensibilità interculturale. Tutte le persone che operano nel settore della scuola, non solo gli insegnanti, dovrebbero pertanto sviluppare la propria competenza interculturale almeno al più elementare dei livelli etnorelativi (Bennett 1993). La buona percezione delle proprie competenze permette alle persone di sviluppare maggiore sensibilità verso gli altri, prestare dunque attenzione ai loro bisogni e alle loro emozioni (Del Vilar 2010). Sebbene sia un percorso non facile e non sempre conduce a risultati sperati, lo sviluppo della sensibilità interpersonale e quindi interculturale risulta una capacità fondamentale nel mondo della scuola e in generale nella società contemporanea.

\section{Riferimenti bibliografici}

Arasaratnam, L. A., e M. L. Doerfel. 2005 «Intercultural Communication Competence: Identifying Key Components from Multicultural Perspectives.» International Journal of Intercultural Relations 29:137-163.

Arzenšek, A. 2012. "Raziskovanje kognitivnih shem kot individualnih ali skupinskih struktur.» Psihološka obzorja 21 (1): 39-49.

Balconi, M. 2008. Neuropsicologia della comunicazione. Milano: Springer.

Bennett, M. J. 1993. «Towards a Developmental Model of Intercultural Sensitivity.» In Education for the Intercultural Experience, a cura di R. M. Paige, 21-71. Yarmouth, M E: Intercultural Press.

Banks, J. A. 2001. «Citizenship Education and Diversity: Implications for Teacher Education.» Journal of Teacher Education 52 (1): 5-16.

Chen, G. M., e W. J. Starosta. 1996. «Intercultural Communication Competence: A Synthesis.» Communication Yearbook 19:353-383.

_ . 1997. «A Review of the Concept of Intercultural Sensitivity.» Human Communication 1:1-16. 
DeJaeghere, J. G., e Y. Cao. 2009. «Development of Intercultural Competence among U S American Teachers.» Intercultural Education 19 (3): 255-268.

Del Vilar, C. P. 2010. «How Savvy Are We? Towards Predicting Intercultural Sensitivity.» Human Communication 13 (3): 197-215.

Gorski, P. C. 2008. «Good Intentions Are Not Enough: A Decolonizing Intercultural Education.» Intercultural Education 19 (6): 515-525.

Hammer, M. R., M. J. Bennett e R. Wiseman. 2003. «Measuring Intercultural Sensitivity: The Intercultural Development Inventory.» International Journal of Intercultural Relations 27:421-443.

Jokikokko, K. 2005. «Interculutrally Trained Finnish Teachers' Conceptions of Diversity and Intercultural Competence.» Intercultural Education 16 (1): 515-525.

Middleton, V. A. 2002. «Increasing Preservice Teacher's Diversity Beliefs and Commitment.» The Urban Review 34 (4):343-361.

Pederson, P. V. 1997. «Intercultural Sensitivity and the Early Adolescent.» Articolo presentato al 77th Annual Conference of the National Council for the Social Studies, Cincinnati, OH, 22-23 novembre.

Segura-Robles, A., e M. E. Parra-González. 2019. "Analysis of Teachers' Intercultural Sensitivity Levels in Multicultural Contexts.» Sustainability 11 (11): 3137.

Spiteri, D. 2017. Multiculturalism, Higher Education and Intercultural Communication: Developing Strengths-Based Narratives for Teaching and Learning. Londra: Macmillan.

Susič, E. 2003. «Socializacija, asimilacija in pravni položaj.» Acta Histriae 11 (2): $237-254$.

Susič, E., e D. Sedmak. 1983. Tiha asimilacija: psihološki vidiki nacionalnega odtujevanja. Trst: Založništvo tržaškega tiska.

Wahyudi, R. 2016. «Intercultural Competence: Multi-dynamic, Intersubjective, Critical and Interdisciplinary Approaches.» In Intercultural Competence in Education, a cura di F. Dervin e Z. Gross, 143-166. Londra: Macmillan.

Zick, A., B. Küpper, e A. Hövermann. 2011. Intollerance, Prejudice and Discrimination: A European Report. Berlin: Friedrich Ebert Stiftung.

Zorman, A., e N. Zudič Antonič. 2014. «Concezione dell'educazione interculturale e sensibilità interculturale tra gli insegnanti.» In Educare alla diversità: ricerca comparativa interdisciplinare tra Italia e Slovenia, a cura di N. Bogatec e N. Zudič Antonič, 105-123. Capodistria: Annales.

- 2019. «Intercultural Sensitivity of Teachers.» Annales: Series Historia et Sociologia 29 (2): 247-258.

Zudič Antonič, N., e A. Zorman. 2004. «Linguistic and Sociocultural Transition in Slovenia: The Italian Ethnic Group, Past and Present.» Transition Studies Review 11 (3): 171-184. 


\section{Vloga medkulturne občutljivosti v sodobni didaktiki jezilkov}

Medkulturna vzgoja mora temeljiti na razvoju vzajemnega razumevanja med ljudmi. Udeleženci $\mathrm{v}$ komunikaciji morajo imeti $\mathrm{v}$ ta namen razvite zmožnosti empatije do sogovorca in zmožnosti konceptualnega vključevanja v miselni svet drugega, da bi skupaj ustvarili pogoje za razumevanje drug drugega. Pri učiteljih, ki nimajo razvite medkulturne občutljivosti in je torej ne zmorejo razvijati pri učencih, niti obsežni programi medkulturne vzgoje v sistemu izobraževanja ne morejo doseči zastavljenih ciljev (Spiteri 2017). Med učitelji in na splošno med ljudmi je pogosto zaznavno protislovje med definicijo medkulturne vzgoje in njenih ciljev na eni strani ter njihovim ravnanjem $\mathrm{v}$ stiku $\mathrm{z}$ osebami, ki jih zaznavajo kot jezikovno, kulturno, etično (Gorski 2008) ali kako drugače različne od sebe. Sistem izobraževanja bi moral omogočiti, da se med seboj zbližajo učeči (in učitelji), ter ustvariti vzdušje, v katerem se izkušnje udeležencev vzajemno bogatijo. $\mathrm{V}$ ta namen je nujno opustiti razmišljanje o ustvarjanju skupne kulture, ki neizogibno predpostavlja potlačenje določenih kultur in poveličevanje drugih. Medkulturno sporazumevanje bi tako moralo izhajati iz individualnosti vsakega učečega in bi moralo biti osnovano na sočutju do drugega. Medkulturna vzgoja mora spodbujati razvoj razumevanja in občutljivosti med ljudmi, ki izhajajo iz različnih jezikovnih in/ali kulturnih okolij, ter se boriti proti družbenemu zatiranju tako, da ljudi seznani in jih ozavesti o tem, kateri mehanizmi v družbi delujejo zatiralno. V nasprotnem primeru je velika verjetnost, da bodo prevzeli vedenjske vzorce, ki jih določa monokulturen pogled na svet, in pri tem izkazovali vidik tiste kulture, ki so mu bili v življenju večinoma izpostavljeni (Spiteri 2017).

Ključne besede: didaktika, medkulturna vzgoja, medkulturna občutljivost, učitelji, učeči

\section{Basis of an Effective Intercultural Education}

Intercultural education must be based on the development of mutual understanding of people. Participants in the communication must thus posess high levels of empathy and the ability to enter conceptually in the world of others in order to generate a mutual comprehension. Theachers with low levels of intercultural sensitivity are not able to develop the sensibility in students, therefore no ammount of intercultural or multicultural education can reach its objectives (Spiteri 2017). Among teachers and among people in general there is often an inconsistency between the definition of intercultural education and its objectives on one hand and their actual conduct in interaction with people they perceive linguistically, culturally, ethnically (Gorski 2008) or some other way different. School should be a place where students (and teachers) come together and benefit from each other. It is essential that for this purpose to abbandon the idea of creating a common 
culture because it glorifies certain cultures and denigrates others. Interculutral communication must be based on each student's individuality as well as on compassion, if it is to promote the comprehension and the senibility among people of different cultural and/or linguistic origin and to fight social oppression by educating or promoting each individual's awareness of what is opressive in their society. Otherwise it is highly probable that they will adopt behavioural schemes determined by a monocultural viewpoint, adapted to the culture they have been exposed to most of their life (Spiteri 2017).

Key words: didactics, intercultural education, intercultural sensibility, teachers, students 